\title{
Publisher's Note: Intertwined order in a frustrated four-leg $t-J$ cylinder [Phys. Rev. B 95, 155116 (2017)]
}

John F. Dodaro, Hong-Chen Jiang, and Steven A. Kivelson (Received 19 September 2017; published 25 September 2017)

DOI: 10.1103/PhysRevB.96.119912

This paper was published online on 12 April 2017 with an error in the Acknowledgments. The second sentence in the Acknowledgments on page 10 should read as "S.A.K. was supported in part by NSF Grant No. DMR 1608055 at Stanford." The Acknowledgments have been corrected as of 18 September 2017. The Acknowledgments are incorrect in the printed version of the journal. 